\title{
Electron, proton and ion induced molecular synthesis and VUV spectroscopy of interstellar molecules in the ice phase
}

\author{
Bhalamurugan Sivaraman ${ }^{1}$, Sohan Jheeta $^{1}$, Nigel Mason ${ }^{1}$, Adam \\ Hunniford $^{2}$, Tony Merrigan ${ }^{2}$, Bob McCullough ${ }^{2}$, Daniele Fulvio ${ }^{3}$, \\ Maria Elisabetta Palumbo ${ }^{3}$, and Marla Moore ${ }^{4}$ \\ ${ }^{1}$ Department of Physics and Astronomy, The Open University, Walton Hall, Milton Keynes, \\ MK7 6AA, UK \\ email: b.sivaraman@open.ac.uk; s.jheeta@open.ac.uk; n.j.mason@open.ac.uk \\ ${ }^{2}$ International Research Centre for Experimental Physics, Queen's University in Belfast \\ email: c.a.hunniford@qub.ac.uk; tmerrigan01@qub.ac.uk; rw.mccullough@qub.ac.uk \\ ${ }^{3}$ Catania Astrophysical Observatory, Catania University \\ email: dfu@oact.inaf.it; elisabetta.palumbo@oact.inaf.it \\ ${ }^{4}$ Cosmic ice lab, NASA, Goddard Space Flight Center \\ email: Marla.H.Moore@nasa.gov
}

\begin{abstract}
Planets and their moons are constantly subjected to irradiation from both their respective planetary magnetospheres and the solar wind. Energetic particles (electrons, protons and ions) in such radiation may induce complex chemistry within the icy mantles of such bodies, producing many organic compounds. Such processes can be simulated in laboratory experiments. In this report we present recent results from experiments exploring both molecular synthesis and the morphology of such ices.

The morphology of any ice may be characterised by IR and Vacuum Ultra-Violet (VUV) spectroscopy. The latter is particularly useful for studying ices in which infrared inactive molecules like oxygen $\left(\mathrm{O}_{2}\right)$ are common. We have shown that oxygen forms dimers in typical planetary ices and that, in contrast to previous analysis, many of the chemical reactions within the ice involve such dimer (and larger cluster) chemistry. We also present the results of a series of experiments that explore electron, proton and ion irradiation on Solar System relevant ices such as carbon dioxide $\left(\mathrm{CO}_{2}\right)$ at different temperatures. Infrared spectra recorded before and after irradiation are used to identify and quantify molecules formed in such irradiation, e. g. ozone. These experiments show that the morphology of the ice plays a critical role in the chemistry.
\end{abstract}

Keywords. Astrochemistry, methods: laboratory, techniques: spectroscopic

\section{Oxygen dimer}

Molecular oxygen $\left(\mathrm{O}_{2}\right)$ has recently been found in interstellar space (Larsson et al. 2007). VUV spectroscopy is considered to be an effective tool to study the properties of this molecule in the ice phase. In our experiments molecular oxygen was deposited at a pressure of $1 \times 10^{-7}$ mbar onto a cold $\mathrm{CaF}_{2}$ window, kept at $\sim 25 \mathrm{~K}$, for different exposure times $(15,20,25$ and 32 second) and VUV spectra were recorded. The feature peaking at $180 \mathrm{~nm}$ was assigned to the $\left(\mathrm{O}_{2}\right)_{2}$, dimer (Mason et al. 2006). The band was very weak when using thin $\mathrm{O}_{2}$ ice layers but grew in intensity with increased deposition to form a thick $\mathrm{O}_{2}$ ice (Figure 1). This suggests that more $\mathrm{O}_{2}$ dimers are formed in thicker ice samples. Therefore, the proportion of dimers and monomers of $\mathrm{O}_{2}$ vary with the ice thickness. 


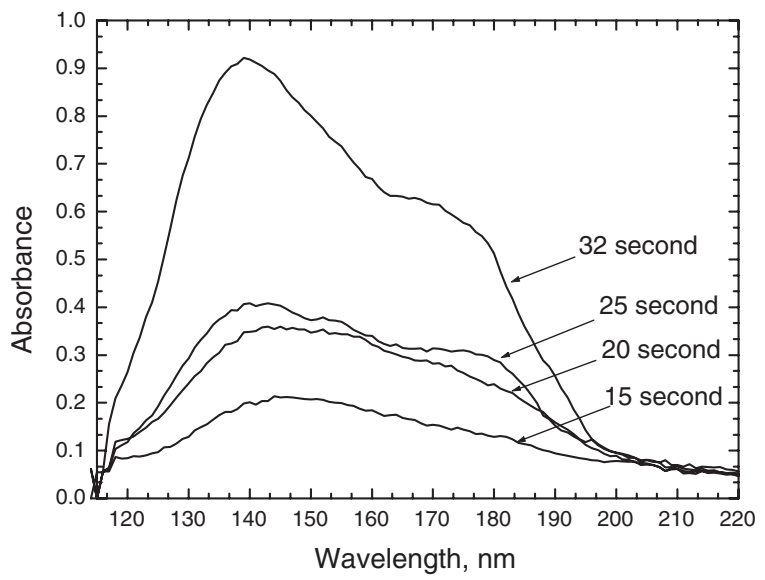

Figure 1. VUV spectra of oxygen ice recorded at different thickness and at $\sim 25 \mathrm{~K}$.

\section{Carbon dioxide}

Laboratory experiments were carried out using $\mathrm{CO}_{2}$ ice to study the processes of importance when magnetospheric ions impinge upon lunar surfaces. Both reactive $\left(\mathrm{D}^{+}, \mathrm{H}^{+}\right)$ and non-reactive $\left(\mathrm{He}^{+}\right)$ions, with energies ranging from $1.5-3 \mathrm{keV}$, were used to irradiate analogues of typical planetary ices. Irradiation was performed at two temperatures $(30$ and $80 \mathrm{~K})$ to explore the effect of ice density and morphology.

In pure $\mathrm{CO}_{2}$ ice, products include $\mathrm{CO}, \mathrm{O}_{3}$ and $\mathrm{CO}_{3}$. In contrast to initial expectations, the largest concentration of $\mathrm{O}_{3}$ was observed at the lower temperature (Figure 2). This is ascribed to $\mathrm{O}$ atoms being more localised in the lower temperature ice (i.e., within a matrix) whilst at higher temperatures they are free to migrate through the ice and more reaction pathways are opened, thereby hindering further $\mathrm{O}_{3}$ production. At $80 \mathrm{~K}$, a small fraction of $\mathrm{O}_{3}$ can also be lost due to sublimation. The same results were observed in recent experiments (Moore et al. 2008) using $10 \mathrm{keV}$ electrons and $0.8 \mathrm{MeV}$ protons on $\mathrm{CO}_{2}$ ices.

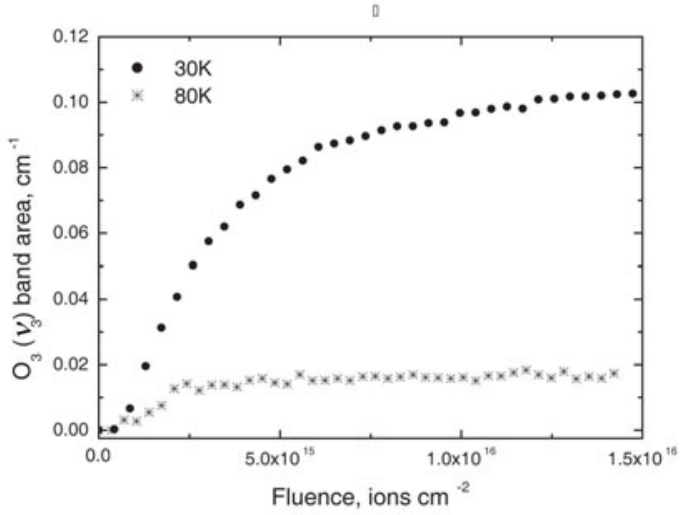

Figure 2. $\mathrm{O}_{3}$ growth during $2.12 \mathrm{keV} \mathrm{D}^{+}$ion irradiation on pure $\mathrm{CO}_{2}$ ice kept at 30 and $80 \mathrm{~K}$.

\section{References}

Larsson, B., et al. 2007, A\&SA, 466, 999

Mason, N. J., et al. 2006, Faraday discussions, 133, 311

Moore, M., Hudson, R. L., Sivaraman, B., \& Mason, N. J. 2008, The formation and destruction of ozone in ices relevant to planetary and satellite surfaces, Abstract submitted to COSPAR 2008 